\title{
Sparse Matrices in Frame Theory
}

\author{
Lemvig, Jakob; Krahmer, Felix; Kutyniok, Gitta
}

\section{Published in:}

Computational Statistics

Link to article, DOI:

10.1007/s00180-013-0446-1

Publication date:

2014

Document Version

Peer reviewed version

Link back to DTU Orbit

Citation (APA):

Lemvig, J., Krahmer, F., \& Kutyniok, G. (2014). Sparse Matrices in Frame Theory. Computational Statistics, 29(3-4), 547-568. https://doi.org/10.1007/s00180-013-0446-1

\section{General rights}

Copyright and moral rights for the publications made accessible in the public portal are retained by the authors and/or other copyright owners and it is a condition of accessing publications that users recognise and abide by the legal requirements associated with these rights.

- Users may download and print one copy of any publication from the public portal for the purpose of private study or research.

- You may not further distribute the material or use it for any profit-making activity or commercial gain

- You may freely distribute the URL identifying the publication in the public portal

If you believe that this document breaches copyright please contact us providing details, and we will remove access to the work immediately and investigate your claim. 


\title{
Sparse Matrices in Frame Theory
}

\author{
Felix Krahmer · Gitta Kutyniok · Jakob \\ Lemvig
}

Received: date / Accepted: date

\begin{abstract}
Frame theory is closely intertwined with signal processing through a canon of methodologies for the analysis of signals using (redundant) linear measurements. The canonical dual frame associated with a frame provides a means for reconstruction by a least squares approach, but other dual frames yield alternative reconstruction procedures. The novel paradigm of sparsity has recently entered the area of frame theory in various ways. Of those different sparsity perspectives, we will focus on the situations where frames and (not necessarily canonical) dual frames can be written as sparse matrices. The objective for this approach is to ensure not only low-complexity computations, but also high compressibility. We will discuss both existence results and explicit constructions.
\end{abstract}

Keywords Dual Frames · Frames · Redundancy $\cdot$ Signal Processing $\cdot$ Tight Frames

F. Krahmer

Universität Göttingen

Institut für Numerische und Angewandte Mathematik

37083 Göttingen, Germany

Tel.: +49-551-3910584

Fax: +49-551-393944

E-mail: f.krahmer@math.uni-goettingen.de

G. Kutyniok

Technische Universität Berlin

Institut für Mathematik

10623 Berlin, Germany

Tel.: +49-30-314-25758

Fax: +49-30-314-21604

E-mail: kutyniok@math.tu-berlin.de

J. Lemvig

Technical University of Denmark

Department of Applied Mathematics and Computer Science

2800 Kgs. Lyngby, Denmark

Tel.: +45-45253065

Fax: $+45-45881399$

E-mail: jakle@dtu.dk 


\section{Introduction}

Efficient signal analysis and processing is one of the great scientific challenges to date due to the ever increasing amounts of data generated in the context of various applications. Recently, sparsity has entered the scene as a novel paradigm allowing the development of highly efficient methodologies for signal processing. The main objectives of this approach are dimension reduction, high compressibility of data, and low-complexity computations.

\subsection{Mathematical Signal Processing}

In mathematical signal processing, the canonical first step is the computation of linear (adaptive or non-adaptive) measurements associated with a carefully designed representation system. To be precise, for a signal $x \in \mathbb{R}^{n}$ and a spanning sequence $\left(\varphi_{i}\right)_{i=1}^{m} \subset \mathbb{R}^{n}$, we compute coefficients $c \in \mathbb{R}^{m}$ by

$$
x \mapsto c:=\Phi^{*} x=\left(\left\langle x, \varphi_{i}\right\rangle\right)_{i=1}^{m} \quad \text { with } \Phi=\left(\varphi_{1}|\ldots| \varphi_{m}\right) \in \mathbb{R}^{n \times m} .
$$

The case when $m=n$ and $\left(\varphi_{i}\right)_{i=1}^{m}$ constitutes an orthonormal basis for $\mathbb{R}^{n}$ is very well studied. More recently, one has considered the following two fundamentally different, more intricate objectives for the transform in (1.1), which go beyond the setting of orthonormal bases. One objective is dimension reduction, i.e., $m<n$, of which compressed sensing is a prominent representative. Another objective is robust analysis, i.e., $m>n$, on which frame theory focuses.

Both cases face the crucial question of whether it is possible to recover $x$ from the measurement coefficients $c=\Phi^{*} x$. In the undercomplete case $(m<n)$, convex optimization is often used, as for instance is the case in compressed sensing (see the survey paper Kutyniok (2013) and the book Eldar and Kutyniok (2012)). In the overcomplete case $(m>n)$, least squares is a typical approach, which frame theory traditionally follows. Thus reconstruction is typically performed by computing

$$
\left(\left(\Phi \Phi^{*}\right)^{-1} \Phi\right) c
$$

In this survey paper, we from now on focus on the overcomplete scenario with the objective of deriving a robust analysis. However, with sparsity entering the picture, as we will see, least squares is not always the preferred method of reconstruction.

\subsection{Frame Theory}

Frame theory - the theory of overcomplete (redundant) Bessel systems - dates back to work by Duffin and Schaeffer (1952) on non-harmonic Fourier series. Its success story in signal processing started in the 1980's with the seminal work by Daubechies et al. (1986). At that time it was recognized that not only can redundancy of $\left(\varphi_{i}\right)_{i=1}^{m}$ ensure robustness of $\left(\left\langle x, \varphi_{i}\right\rangle\right)_{i=1}^{m}$ against noise or erasures, but the restrictions of forming an orthonormal basis are often too strong for the construction of many systems. Since the groundbreaking work by Daubechies et al. (1986) was published, frames 
have become a key notion in signal processing. It should be emphasized that frame theory is not only widely used in the finite dimensional setting (cf. Casazza and Kutyniok (2013)), but also in infinite dimensions. However, in this survey paper we focus on finite frames.

In signal processing with frames, three main steps can be identified. The first step is decomposition with frames, which is performed by the mapping in (1.1). Main objectives for this step are the design of frames $\Phi$ that can easily be stored and that allow for a low-complexity computation of $\Phi^{*} x$.

The second step is the analysis of the signal based on the frame coefficients $c=\Phi^{*} x$. Depending on the processing goal (feature detection, inpainting, transmission, etc.), the frame $\Phi$ needs to be designed accordingly, for instance, by encoding the needed features in the large coefficients. Another main issue in signal processing is linear or non-linear operators $A: \mathbb{R}^{m} \rightarrow \mathbb{R}^{m}$ applied to the measurements $\Phi^{*} x$ during the analysis or transmission process. Examples for such operators are erasure operators associated with a diagonal matrix with diagonal entries either 0 or 1 , thresholding operators which set to zero all entries smaller (in absolute value) than a given value, or the operator which takes the absolute value of each entry leading to the problem of phase retrieval. Hence one goal in such settings is to minimize the error $\max _{\|x\|_{2}=1}\left\|x-\left(\left(\Phi \Phi^{*}\right)^{-1} \Phi\right) A \Phi^{*} x\right\|_{2}$.

The third step consists in reconstruction of the original or manipulated signal, which is for instance done by (1.2), where $c$ could be either the original or modified frame coefficients depending on the analysis step. Again, low-complexity computations are one concern. As a consequence, it is often desirable to choose a frame $\Psi$, a so-called dual frame, different from $\left(\Phi \Phi^{*}\right)^{-1} \Phi$ for reconstruction. The reconstruction is then performed by computing $\Psi_{c}$. This alternative reconstruction approach leads to design questions regarding the dual frame $\Psi$.

The above considerations show the richness of the tasks in frame theory, which is also reflected in the large number of commonly used frame constructions, e.g., equiangular frames, harmonic frames, Gabor frames, wavelet frames, and shearlet frames.

\subsection{Desideratum: Sparsity}

Sparsity has become an important paradigm in both numerical linear algebra and signal processing. The sparsity of a vector $x=\left(x_{1}, \ldots, x_{n}\right)^{t} \in \mathbb{R}^{n}$ is measured by

$$
\|x\|_{0}:=\#\left\{i \in\{1, \ldots, n\}: x_{i} \neq 0\right\},
$$

and $x$ is called $k$-sparse, if $\|x\|_{0} \leq k$. Along the same lines, sparsity of a matrix means that many of the matrix entries vanish, i.e., the quantity

$$
\left.\|\Phi\|_{0}:=\#\left\{(i, j): \varphi_{i, j} \neq 0\right\}, \quad \text { for } \Phi=\varphi_{i, j}\right] \in \mathbb{R}^{n \times m}
$$

being small. Sparsity nowadays plays two conceptually very different roles. On the one hand, sparse representations guarantee efficient storage and processing of data. For example, multiplying a vector with a sparse matrix requires less operations and 
the sparse representation of a signal can be directly used for efficient compression. On the other hand, sparse representations epitomize structural simplicity. In fact, many problems in signal processing are intrinsically ill-posed, so only such structural assumptions on the solution make accurate and stable solutions possible. Key examples are in the theory of compressed sensing as introduced in parallel by Donoho (2006) and Candès et al. (2006) in 2006. In this context, also weaker versions of sparsity such as compressibility or the behavior of the error of the best $k$-term approximation are frequently used.

Both these usages of sparsity have direct connections to frame theory. For the first usage, this connection can be made in two different ways. Firstly, one can aim to design frames and dual frames to form sparse matrices themselves, with the goal of an efficient decomposition and reconstruction process. Secondly, the additional freedom coming from the redundancy when working with frame representations rather than basis representations can allow the derivation of sparser representations of a vector. The second usage appears in frame theory as a design question for the measurement matrix in compressed sensing, which computes $\Phi x$ for some $x$ in the higher dimensional space $\mathbb{R}^{m}$.

In this survey, we focus on the very first connection, that is, sparsity of the frame and dual frame matrices as a means to ensure a more efficient decomposition and reconstruction process. However, the inverse problems viewpoint on sparsity also plays an important role, as finding sparse frames under certain constraints has many structural similarities to finding sparse solutions to inverse problems.

Frames as sparse matrices were first analyzed in 2011 by Casazza et al. (2011b), whereas sparse duals were first considered and discussed independently by Krahmer et al. (2013) and Li et al. (2013). For both situations, results on the optimal sparsity were derived, existence results were proven, and algorithms provided.

\subsection{Outline}

We provide an introduction to frame theory in Section 2. In Section 3, we then focus on sparse frames and discuss optimality results, existence results, and explicit constructions. Similar considerations will be undertaken in Section 4, but this time focusing on sparsity of the associated dual frames.

\section{Basics of Frame Theory}

We start with reviewing the basic definitions and notations of frame theory, which will be used in the sequel. For more details and additional information, we refer the reader to the books by Christensen (2008) and Casazza and Kutyniok (2013).

\subsection{Frames}

For simplicity we present the definitions and most of the results in this survey paper solely for the real case. The only exceptions are results on frames related to Fourier 
and Gabor expansions in Section 4 as such frames have particularly efficient representation over $\mathbb{C}$. Independent of this distinction, we remark that all results in this survey paper also hold for the complex case.

A frame is a family of vectors which ensures stability of the map introduced in (1.1). The precise definition is as follows.

Definition 2.1 A family of vectors $\left(\varphi_{i}\right)_{i=1}^{m}$ in $\mathbb{R}^{n}$ is called a frame for $\mathbb{R}^{n}$, if there exist constants $0<A \leq B<\infty$ such that

$$
A\|x\|^{2} \leq \sum_{i=1}^{m}\left|\left\langle x, \varphi_{i}\right\rangle\right|^{2} \leq B\|x\|^{2} \quad \text { for all } x \in \mathbb{R}^{n}
$$

The constants $A$ and $B$ are called lower and upper frame bound for the frame, respectively. If $A=B$ is possible in (2.1) then $\left(\varphi_{i}\right)_{i=1}^{m}$ is called an $A$-tight frame. If $A=B=1$ it is called a Parseval frame, as in this case, (2.1) is a direct analog to Parseval's identity. If there exists a constant $c$ such that $\left\|\varphi_{i}\right\|=c$ for all $i=1,2, \ldots, m$, then $\left(\varphi_{i}\right)_{i=1}^{m}$ is an equal-norm frame. If $c=1,\left(\varphi_{i}\right)_{i=1}^{m}$ is a unit-norm frame. Finally, the values $\left(\left\langle x, \varphi_{i}\right\rangle\right)_{i=1}^{m}$ are called the frame coefficients of the vector $x$ with respect to the frame $\left(\varphi_{i}\right)_{i=1}^{m}$.

For a given frame $\Phi=\left(\varphi_{i}\right)_{i=1}^{m}$ and a fixed orthonormal basis $\left(e_{j}\right)_{j=1}^{n}$, we let $\Phi$ denote the $n \times m$ frame matrix, whose $i$ th column is the coefficient vector of $\varphi_{i}$. Note that with a slight abuse of notation, we denote both the frame and the corresponding frame matrix by $\Phi$. The condition (2.1) for a frame then reads

$$
A\|x\|^{2} \leq\left\|\Phi^{*} x\right\|^{2} \leq B\|x\|^{2} \text { for all } x \in \mathbb{R}^{n}
$$

Several useful observations are combined in the following result. Since the proofs are straightforward, we leave them to the reader.

Lemma 2.2 Let $\left(\varphi_{i}\right)_{i=1}^{m}$ be a family of vectors in $\mathbb{R}^{n}$.

(i) If $\left(\varphi_{i}\right)_{i=1}^{m}$ is an orthonormal basis, then $\left(\varphi_{i}\right)_{i=1}^{m}$ is a Parseval frame. The converse is not true in general.

(ii) $\left(\varphi_{i}\right)_{i=1}^{m}$ is a unit-norm Parseval frame if and only if it is an orthonormal basis.

Simple examples of equal-norm Parseval frames for $m>n$ are given by any concatenation of orthonormal bases. A standard example for an equal-norm Parseval frame which does not have this structure is the frame in $\mathbb{R}^{2}$ given by

$$
\left\{\sqrt{\frac{2}{3}}\left(\begin{array}{l}
0 \\
1
\end{array}\right), \sqrt{\frac{2}{3}}\left(\begin{array}{c}
\frac{\sqrt{3}}{2} \\
-\frac{1}{2}
\end{array}\right), \sqrt{\frac{2}{3}}\left(\begin{array}{c}
-\frac{\sqrt{3}}{2} \\
-\frac{1}{2}
\end{array}\right)\right\}
$$

Due to the shape of its vectors in $\mathbb{R}^{2}$, this frame is typically called the Mercedes-Benz frame. 
2.2 The Frame Operator

Given a frame $\left(\varphi_{i}\right)_{i=1}^{m}$, its signal processing performance is determined by the following three operators. We remark that the first operator was already introduced in (1.1).

Definition 2.3 Let $\Phi=\left(\varphi_{i}\right)_{i=1}^{m}$ be a frame in $\mathbb{R}^{n}$.

(i) The associated analysis operator embeds $\mathbb{R}^{n}$ into $\mathbb{R}^{m}$ and is given by

$$
x \mapsto \Phi^{*} x=\left(\left\langle x, \varphi_{i}\right\rangle\right)_{i=1}^{m}, \quad x \in \mathbb{R}^{n} .
$$

(ii) The associated synthesis operator is defined to be the adjoint of the analysis operator, thus mapping $\mathbb{R}^{m}$ back to $\mathbb{R}^{n}$. Consequently, it is given by

$$
c \mapsto \Phi c=\sum_{i=1}^{m} c_{i} \varphi_{i}, \quad c=\left(c_{i}\right)_{i=1}^{m} \in \mathbb{R}^{m}
$$

(iii) The associated frame operator is the map $S: \mathbb{R}^{n} \rightarrow \mathbb{R}^{n}$ defined as the composition of these two operators, that is,

$$
S x:=\Phi \Phi^{*} x=\sum_{i=1}^{m}\left\langle x, \varphi_{i}\right\rangle \varphi_{i}, \quad x \in \mathbb{R}^{n} .
$$

The following result is well-known; a proof can be found for example in Christensen (2008), In the sequel, $I_{n}$ will always denote the identity matrix on $\mathbb{R}^{n}$.

Theorem 2.4 The frame operator $S$ of a frame $\left(\varphi_{i}\right)_{i=1}^{m}$ for $\mathbb{R}^{n}$ with frame bounds $A$ and $B$ is a positive, self-adjoint invertible operator satisfying

$$
A \cdot I_{n} \leq S \leq B \cdot I_{n}
$$

\subsection{Reconstruction Strategy}

We next aim to reconstruct the original signal from the image under the analysis operator. This can be achieved by application of a linear operator by using Theorem 2.4 as the following result shows.

Theorem 2.5 Let $\Phi=\left(\varphi_{i}\right)_{i=1}^{m}$ be a frame for $\mathbb{R}^{n}$ with frame operator $S$. Then, for every $x \in \mathbb{R}^{n}$, we have

$$
x=S^{-1} S x=\left(\left(\Phi \Phi^{*}\right)^{-1} \Phi\right)\left(\Phi^{*} x\right)=\sum_{i=1}^{m}\left\langle x, \varphi_{i}\right\rangle S^{-1} \varphi_{i}
$$

This is the well-known least squares reconstruction. Due to the redundancy of the frame, other matrices $\Psi \in \mathbb{R}^{n \times m}$ exist which satisfy the reconstruction condition $\Psi \Phi^{*}=I_{n}$. For those, we have the following terminology. 
Definition 2.6 Let $\Phi=\left(\varphi_{i}\right)_{i=1}^{m}$ be a frame for $\mathbb{R}^{n}$ with frame operator $S$. Then the system $\left(S^{-1} \varphi_{i}\right)_{i=1}^{m}=\left(\Phi \Phi^{*}\right)^{-1} \Phi$ is called the canonical dual frame. In general, every frame $\Psi=\left(\psi_{i}\right)_{i=1}^{m}$ for $\mathbb{R}^{n}$ satisfying

$$
x=\Psi \Phi^{*} x=\sum_{i=1}^{m}\left\langle x, \varphi_{i}\right\rangle \psi_{i}, \quad x \in \mathbb{R}^{n}
$$

is referred to as an (alternate) dual frame for $\Phi$.

The set of all dual frames can be explicitly expressed. This classical result is due to $\mathrm{Li}(1995)$.

Proposition 2.7 (Li (1995)) Let $\Phi=\left(\varphi_{i}\right)_{i=1}^{m}$ be a frame for $\mathbb{R}^{n}$. Then, every dual frame $\Psi=\left(\psi_{i}\right)_{i=1}^{m}$ for $\Phi$ is of the form

$$
\Psi=\left(\Phi \Phi^{*}\right)^{-1} \Phi+L\left(I_{m}-\Phi^{*}\left(\Phi \Phi^{*}\right)^{-1} \Phi\right), \quad \text { where } L \in \mathbb{R}^{n \times m}
$$

We remark that the canonical dual frame plays no special role in (2.2) in the sense that, if $\widetilde{\Psi}$ is just some dual, then all duals are obtained by

$$
\Psi=\widetilde{\Psi}+L\left(I_{m}-\Phi^{*} \widetilde{\Psi}\right)
$$

where $L \in \mathbb{R}^{n \times m}$.

If $\Phi$ forms an $A$-tight frame, then by Theorem 2.4, the frame operator is a multiple of the identity. This leads to the reconstruction formula

$$
x=A^{-1} \Phi\left(\Phi^{*} x\right)=A^{-1} \sum_{i=1}^{m}\left\langle x, \varphi_{i}\right\rangle \varphi_{i}
$$

In this case, the canonical dual frame coincides with $A^{-1} \Phi$. This shows that from a signal processing perspective, tight frames are highly desirable.

\subsection{Expansion in Frames}

From Theorem 2.4 we can also deduce a different representation formula, which can be regarded as an expansion of $x$ in terms of the frame $\left(\varphi_{i}\right)_{i=1}^{m}$.

Theorem 2.8 Let $\Phi=\left(\varphi_{i}\right)_{i=1}^{m}$ be a frame for $\mathbb{R}^{n}$ with frame operator $S$. Then, for every $x \in \mathbb{R}^{n}$, we have

$$
x=S S^{-1} x=\left(\Phi \Phi^{*}\right)\left(\Phi \Phi^{*}\right)^{-1} x=\Phi\left(\left(\Phi \Phi^{*}\right)^{-1} \Phi\right)^{*} x=\sum_{i=1}^{m}\left\langle x, S^{-1} \varphi_{i}\right\rangle \varphi_{i}
$$

The specifically chosen sequence of coefficients $\left(\left(\Phi \Phi^{*}\right)^{-1} \Phi\right)^{*} x$ is the one being minimal in the $\ell_{2}$ norm among all coefficient sequences. 
Proposition 2.9 Let $\Phi=\left(\varphi_{i}\right)_{i=1}^{m}$ be a frame for $\mathbb{R}^{n}$ with frame operator $S$, and let $x \in \mathbb{R}^{n}$. If $c=\left(c_{i}\right)_{i=1}^{m}$ are scalars such that $x=\Phi c=\sum_{i=1}^{m} c_{i} \varphi_{i}$, then

$$
\begin{aligned}
\|c\|_{2}^{2} & =\left\|\left(\left(\Phi \Phi^{*}\right)^{-1} \Phi\right)^{*} x\right\|_{2}^{2}+\left\|c-\left(\left(\Phi \Phi^{*}\right)^{-1} \Phi\right)^{*} x\right\|_{2}^{2} \\
& =\sum_{i=1}^{m}\left|\left\langle x, S^{-1} \varphi_{i}\right\rangle\right|^{2}+\sum_{i=1}^{m}\left|c_{i}-\left\langle x, S^{-1} \varphi_{i}\right\rangle\right|^{2} .
\end{aligned}
$$

For a proof of Proposition 2.9 we again refer to Christensen (2008). Proposition 2.9 immediately implies that for any sequence $c=\left(c_{i}\right)_{i=1}^{m}$ satisfying $x=\sum_{i=1}^{m} c_{i} \varphi_{i}$, we have

$$
\sum_{i=1}^{m}\left|\left\langle x, S^{-1} \varphi_{i}\right\rangle\right|^{2}=\left\|\left(\left(\Phi \Phi^{*}\right)^{-1} \Phi\right)^{*} x\right\|_{2}^{2}<\|c\|_{2}^{2}
$$

unless $c=\left(\left(\Phi \Phi^{*}\right)^{-1} \Phi\right)^{*} x$.

\subsection{Construction of Tight Frames}

As already noted, due to their advantageous reconstruction properties, it is particularly desirable to construct tight frames. We distinguish between two different cases: the situation where a given frame shall be modified to become a tight frame, and the situation where one aims to design a frame satisfying certain desirable properties.

In case a frame is already given, there exists a very straightforward way to modify it in order to turn the frame into a Parseval frame.

Proposition 2.10 Let $\Phi=\left(\varphi_{i}\right)_{i=1}^{m}$ be a frame for $\mathbb{R}^{n}$ with frame operator $S$. Then

$$
\left(S^{-1 / 2} \varphi_{i}\right)_{i=1}^{m}=\left(\Phi \Phi^{*}\right)^{-1 / 2} \Phi
$$

forms a Parseval frame.

Proof This follows from

$$
\left(\Phi \Phi^{*}\right)^{-1 / 2} \Phi\left(\left(\Phi \Phi^{*}\right)^{-1 / 2} \Phi\right)^{*}=\left(\Phi \Phi^{*}\right)^{-1 / 2} \Phi \Phi^{*}\left(\Phi \Phi^{*}\right)^{-1 / 2}=I_{n}
$$

and the definition of a Parseval frame.

However, various properties of the frame such as the direction of the frame vectors is destroyed during the process. A much more simple procedure, which can also be regarded as preconditioning by a diagonal matrix, is to scale each frame vector to generate a Parseval frame. Characterizing conditions - also of geometric type - for a frame to be scalable in this sense were obtained by Kutyniok et al. (2013).

If no frame is given, but only the dimension of the space $n$, and the number of frame vectors $m$, there are also explicit algorithms to construct a corresponding tight frame. A specific algorithm which accomplishes this goal will be described in Section 3.1. The algorithm is of special relevance to this paper as the frames constructed are particularly sparse. 


\section{Sparse Frames}

In this section, we will study our guiding problem in the most general context: How sparse can a frame be when the embedding and ambient dimensions $n$ and $m$ are given? We will ask this question in regard to the following definition of a sparse frame.

Definition 3.1 Let $\left(e_{j}\right)_{j=1}^{n}$ be an orthonormal basis for $\mathbb{R}^{n}$. Then a frame $\left(\varphi_{i}\right)_{i=1}^{m}$ for $\mathbb{R}^{n}$ is called $k$-sparse with respect to $\left(e_{j}\right)_{j=1}^{n}$, if, for each $i \in\{1, \ldots, m\}$, there exists $J_{i} \subseteq\{1, \ldots, n\}$ such that

$$
\varphi_{i} \in \operatorname{span}\left\{e_{j}: j \in J_{i}\right\}
$$

and

$$
\sum_{i=1}^{n}\left|J_{i}\right|=k
$$

Note that according to this definition, a frame being $k$-sparse is the same as the associated frame matrix having at most $k$ non-vanishing entries, hence this definition is in line with (1.3). The main goal of this section will be to find optimally sparse frames in the sense of the following definition.

Definition 3.2 Let $\left(e_{j}\right)_{j=1}^{n}$ be an orthonormal basis for $\mathbb{R}^{n}$, let $\mathscr{F}$ be a class of frames for $\mathbb{R}^{n}$ consisting of $m$ frame vectors, and let $\left(\varphi_{i}\right)_{i=1}^{m} \in \mathscr{F}$. Then $\left(\varphi_{i}\right)_{i=1}^{m}$ is called optimally sparse in $\mathscr{F}$ with respect to $\left(e_{j}\right)_{j=1}^{n}$, if there exists $k_{1} \in \mathbb{N}$ such that $\left(\varphi_{i}\right)_{i=1}^{m}$ is $k_{1}$-sparse with respect to $\left(e_{j}\right)_{j=1}^{n}$ and there does not exist a frame $\left(\psi_{i}\right)_{i=1}^{m} \in \mathscr{F}$ which is $k_{2}$-sparse with respect to $\left(e_{j}\right)_{j=1}^{n}$ for any $k_{2}<k_{1}$.

Without any additional constraints, an optimally sparse frame will always be given by the canonical basis extended by zero vectors. However, since the first $n$ frame coefficients of a vector will carry all the information, the frame representation cannot be called redundant. A way to circumvent this issue is to require the frame vectors to be unit norm, i.e., $\left\|\varphi_{i}\right\|_{2}=1$. This normalization ensures that the frame coefficients carry equal information. Even then an optimally sparse frame is easily found by extending the canonical basis by $m-n$ copies of the first (or any other) basis vector. The resulting frame, however, has another drawback: Its redundancy is distributed very unevenly. In the direction of the repeated frame vector it is indeed very redundant, whereas in all other directions the frame is not redundant at all. The spatial distribution of the redundancy is studied by Bodmann et al. (2011), and the authors show that this is reflected in the spectrum of the frame operator. Consequently, we will fix the spectrum of the frame operator as an additional constraint.

Arguably the most natural case is that the desired redundancy is evenly distributed, which corresponds to a spectrum consisting of a single point and hence a tight frame. Thus our main focus in the remainder of this section will be on the class of frames

$$
\mathscr{F}(n, m)=\left\{\Phi \in \mathbb{R}^{n \times m}: \Phi \text { is a unit norm, tight frame }\right\} .
$$

In Remark 3.8 we will, however, briefly discuss extensions to more general spectra. 


\subsection{Spectral Tetris - an Algorithm to Construct Sparse Tight Frames}

Frames in $\mathscr{F}(n, m)$ are characterized by having a frame matrix with orthogonal rows with norm $\sqrt{m / n}$ and columns with norm one. Constructing a sparse unit norm, tight frame hence reduces to satisfying these constraints using as few entries of the matrix as possible and setting the remaining entries to zero. The objective of the spectral tetris algorithm, as introduced by Casazza et al. (2011a), is to accomplish these goals in a greedy fashion, determining the frame matrix entries subsequently in a recursive way. Its only requirement is that $m / n \geq 2$; for such dimension pairings it constructs a unit-norm tight frame $\left(\varphi_{i}\right)_{i=1}^{m}$ for $\mathbb{R}^{n}$. The frame bound then automatically equals $m / n$. The detailed steps are provided in Algorithm 1. We remark that an extension to arbitrary spectra of the frame operator is described in Calderbank et al. (2011).

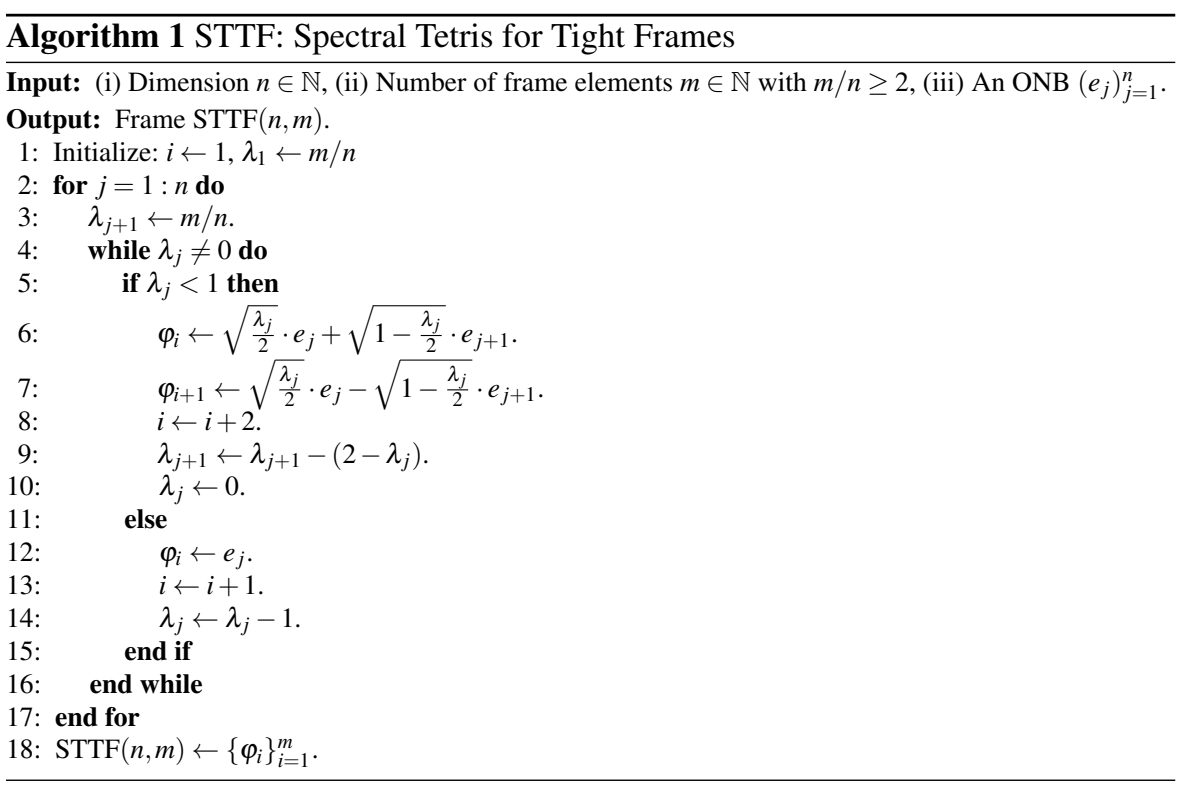

By construction, the frames resulting from Algorithm 1 will always be rather sparse. Indeed, the two cases which the algorithm distinguishes in the if-else statement correspond to adding a column with either one non-vanishing entry or two nonvanishing entries. Thus in any case, the frame has sparsity at most $2 m$, which is small compared to the total number of $m n$ matrix entries. The intrinsic sparsity of the algorithmic procedure is best visualized by the spectral tetris frame given in Example 3.7 below.

Our goal for the remainder of this section is first to find a lower bound for the optimal sparsity within the class $\mathscr{F}(n, m)$ and then to show that the spectral tetris frames, when they exist, in fact obtain this lower bound. As we will see in the following subsection, a factor that determines the optimal sparsity and that is consequently a key proof ingredient is whether the dimension pairing allows for block decompositions. 


\subsection{Block Decompositions of Frame Matrices}

Recall that Algorithm 1 returns a frame consisting only of 1-sparse and 2-sparse vectors. Hence the only way one can expect to find an even sparser frame would be to have fewer 2-sparse vectors than the output of Algorithm 1. So it is logical to ask for the minimal number of 2-sparse vectors that a frame of given embedding and ambient dimensions must have. Spectral tetris frames contain at most $2 n-2$ frame vectors which are 2 -sparse, but can contain less. In the latter case, the frame matrix has a block decomposition of order at least 2 in the sense of the following definition.

Definition 3.3 Let $n, m>0$, and let $\left(\varphi_{i}\right)_{i=1}^{m}$ be a frame for $\mathbb{R}^{n}$. Then we say that the frame matrix $\Phi=\left(\varphi_{i}\right)_{i=1}^{m}$ has block decomposition of order $\eta$, if there exists a partition $\{1, \ldots, m\}=I_{1} \cup \ldots \cup I_{\eta}$ such that, for any $i_{1} \in I_{\ell_{1}}$ and $i_{2} \in I_{\ell_{2}}$ with $\ell_{1} \neq \ell_{2}$, we have $\operatorname{supp} \varphi_{i_{1}} \cap \operatorname{supp} \varphi_{i_{2}}=\emptyset$ and $\eta$ is maximal.

The following result now connects the block decomposition order of a frame matrix with the greatest common divisor of the dimension and the number of frame vectors.

Proposition 3.4 (Casazza et al. (2011b)) Let $m>n>0$ and $\Phi=\left(\varphi_{i}\right)_{i=1}^{m} \in \mathscr{F}(n, m)$. Then the frame matrix $\Phi$ has block decomposition of order at most $\operatorname{gcd}(n, m)$.

Proof Assume $\left(\varphi_{i}\right)_{i=1}^{m} \in \mathscr{F}(n, m)$ has block decomposition of order $\eta$, and let a corresponding partition be given by $\{1, \ldots, m\}=I_{1} \cup \ldots \cup I_{\eta}$. For each $\ell=1, \ldots, \eta$, let $S_{\ell}$ be the common support set of the vectors $\left(\varphi_{i}\right)_{i \in I_{\ell}}$. In other words, we have $k \in S_{\ell}$ if and only if $k \in \operatorname{supp} \varphi_{i}$ for some $i \in I_{\ell}$. Further, let $r_{k}$ denote the $k$ th row of the frame matrix of $\left(\varphi_{i}\right)_{i=1}^{m}$; note that $\left\|r_{k}\right\|^{2}=\frac{m}{n}$ as the frame is tight. Then $S_{1}, \ldots, S_{\eta}$ is a partition of $\{1, \ldots, n\}$ and, for every $\ell=1, \ldots, \eta$, we have by the fact that $\left(\varphi_{i}\right)_{i=1}^{m}$ consists of unit norm vectors and by our choice of $I_{\ell}$ and $S_{\ell}$ that

$$
\# I_{\ell}=\sum_{k \in I_{\ell}}\left\|\varphi_{k}\right\|^{2}=\sum_{k \in S_{\ell}}\left\|r_{k}\right\|^{2}=\sum_{k \in S_{\ell}} \frac{m}{n}=\# S_{\ell} \frac{m}{n} .
$$

The second equality holds since we, after permutation of the columns, can write the frame matrix of $\left(\varphi_{i}\right)_{i=1}^{m}$ as $\Phi=\left[\Phi_{1}, \ldots, \Phi_{\eta}\right]$, where $\Phi_{\ell}$ has zero entries except on the rows indexed by $I_{\ell}$ and the columns indexed by $S_{\ell}$, for $\ell=1, \ldots, \eta$. As each $\# I_{\ell}$ is an integer, one must have $\# S_{\ell} \geq \frac{n}{\operatorname{gcd}(n, m)}$ for all $\ell$, and hence we obtain $\eta \leq \frac{n}{\min n_{\ell} S_{\ell}} \leq$ $\operatorname{gcd}(n, m)$.

\subsection{A Lower Bound for Sparsity within $\mathscr{F}(n, m)$}

The main result of this section, Theorem 3.5, is a lower bound on the achievable sparsity of frames in the class $\mathscr{F}(n, m)$. With Theorem 3.6 in the next section, we will see that this lower bound is actually realizable for any $n, m \in \mathbb{N}$ in the range $m \geq 2 n$. 
Theorem 3.5 (Casazza et al. (2011b)) Let $m>n>0$. Suppose that $\left(\varphi_{i}\right)_{i=1}^{m} \in \mathscr{F}(n, m)$. Then

$$
\|\Phi\|_{0} \geq m+2(n-\operatorname{gcd}(n, m))
$$

where $\Phi$ is the frame matrix of $\left(\varphi_{i}\right)_{i=1}^{m}$ with respect to any orthonormal basis.

Proof Let $\Phi$ denote the frame matrix of a frame in $\mathscr{F}(n, m)$ with respect to a fixed orthonormal basis. For the sake of brevity, in the sequel we will use the phrase that two rows of $\Phi$ have overlap of size $k$, if the intersection of their supports is a set of size $k$. Note that, since the rows of $\Phi$ are orthogonal, it is not possible that two rows of $\Phi$ have overlap of size one.

We first consider the case where $\operatorname{gcd}(n, m)=1$. Pick an arbitrary row $r_{1}$ of $\Phi$. Since, by Proposition 3.4, $\Phi$ has block decomposition of order one, there exists a row $r_{2}$ whose overlap with $r_{1}$ is of size two or greater. Similarly, there has to exist a row different from $r_{1}$ and $r_{2}$ which has overlap of size two or greater with either $r_{1}$ or $r_{2}$. Iterating this procedure will provide an order $r_{1}, r_{2}, \ldots r_{n}$ such that, for each row $r_{j}$, there exists some $k<j$ such that $r_{j}$ has overlap of size two or greater with $r_{k}$. Since all columns in $\Phi$ are of norm one, for each column $v$, there exists a minimal $j$ for which the $r_{j}$ th entry of the vector $v$ is non-zero. This yields $m$ non-zero entries in $\Phi$. In addition, each row $r_{2}$ through $r_{n}$ has at least two non-zero entries coming from the overlap, which are different from the $m$ entries just accounted for, since these entries cannot be the non-zero entries of minimal index of a column due to the overlap with a previous row. This sums up to a total of at least $2(n-1)$ non-zero coefficients. Consequently, the frame matrix has at least $m+2(n-1)$ non-zero entries.

We now consider the case where $\operatorname{gcd}(n, m)=\eta>1$. By Proposition 3.4, the matrix $\Phi$ has block decomposition of order at most $\eta$. Performing the same construction as above, we see that there exist at most $\eta$ rows $r_{j}$ (including the first one) which do not have overlap with a row $r_{k}$ for $k<j$. Thus the frame matrix $\Phi$ must at least contain $m+2(n-\eta)$ non-zero entries.

\subsection{Optimally Sparse Unit Norm Tight Frames}

Having set the benchmark, we now prove that frames constructed by spectral tetris in fact meet the optimal sparsity rate. For this, we remind the reader that the tight spectral tetris frame as constructed by $\operatorname{Algorithm} 1$ is denoted by $\operatorname{STTF}(n, m)$.

Theorem 3.6 (Casazza et al. (2011b)) Let $m \geq 2 n>0$. Let $\left(e_{j}\right)_{j=1}^{n}$ be an orthonormal basis for $\mathbb{R}^{n}$. Then the frame $\operatorname{STTF}(n, m)$ constructed using $\left(e_{j}\right)_{j=1}^{n}$ is optimally sparse in $\mathscr{F}(n, m)$ with respect to $\left(e_{j}\right)_{j=1}^{n}$. That is, if $\Phi$ is the frame matrix of $\operatorname{STTF}(n, m)$ with respect to $\left(e_{j}\right)_{j=1}^{n}$, then

$$
\|\Phi\|_{0}=m+2(n-\operatorname{gcd}(n, m)) .
$$

Proof Let $\left(\varphi_{i}\right)_{i=1}^{m}$ be the frame $\operatorname{STTF}(n, m)$, and let $\Phi$ be the frame matrix of $\left(\varphi_{i}\right)_{i=1}^{m}$ with respect to $\left(e_{j}\right)_{j=1}^{n}$. We start by showing that $\Phi$ has block decomposition of order $\eta=\operatorname{gcd}(n, m)$. For this, we set $n_{0}=m_{0}=0$ and $n_{j}=j \frac{n}{\eta}, m_{j}=j \frac{m}{\eta}, 1 \leq j \leq \eta$; in particular this entails $k_{\eta}=n$ and $m_{\eta}=m$. 
As $\frac{m}{n}=\frac{m_{1}}{n_{1}}$, the first $n_{1}$ entries of the first $m_{1}$ coefficient vectors resulting from $\operatorname{STTF}\left(n_{1}, m_{1}\right)$ and $\operatorname{STTF}(n, m)$ coincide; indeed, the corresponding steps are identical. Continuing the computation of $\operatorname{STTF}(n, m)$ will set the remaining entries of the first $m_{1}$ vectors and also the first $n_{1}$ entries of the remaining vectors to zero. Thus, any of the first $n_{1}$ vectors has disjoint support from any of the vectors constructed later on. Repeating this argument for $n_{2}$ until $n_{\eta}$, we obtain that $\Phi$ has a block decomposition of order $\eta$; the corresponding partition of the frame vectors being

$$
\bigcup_{i=1}^{\eta}\left\{\varphi_{m_{i-1}+1}, \ldots, \varphi_{m_{i}}\right\} .
$$

To compute the number of non-zero entries in $\Phi$, we let $i \in\{1, \ldots, \eta\}$ be arbitrarily fixed and compute the number of non-zero entries of the vectors $\varphi_{m_{i-1}+1}, \ldots, \varphi_{m_{i}}$. Spectral tetris ensures that the support of each of the rows $n_{i-1}+1$ up to $n_{i}-1$ intersects the support of the subsequent row on a set of size two, as otherwise an additional block would be created. Thus, there exist $2\left(n_{i}-n_{i-1}-1\right)$ frame vectors with two non-zero entries. The remaining $\left(m_{i}-m_{i-1}\right)-2\left(n_{i}-n_{i-1}-1\right)$ frame vectors will have only one entry, yielding a total number of $\left(m_{i}-m_{i-1}\right)+2\left(n_{i}-n_{i-1}-1\right)$ non-zero entries in the vectors $\varphi_{m_{i-1}+1}, \ldots, \varphi_{m_{i}}$. is

Summarizing, the total number of non-zero entries in the frame vectors of $\left(\varphi_{i}\right)_{i=1}^{m}$

$$
\begin{aligned}
\sum_{i=1}^{\eta}\left(m_{i}-m_{i-1}\right)+2\left(n_{i}-n_{i-1}-1\right) & =\left(\sum_{i=1}^{\eta}\left(m_{i}-m_{i-1}\right)\right)+2\left(n_{\eta}-\left(\sum_{i=1}^{\eta} 1\right)\right) \\
& =m+2(n-\eta),
\end{aligned}
$$

which, by Theorem 3.5 , is the optimal sparsity.

The following example shows that an optimally sparse frame from $\mathscr{F}(n, m)$ is, in general, not unique.

Example 3.7 Let $n=4$ and $m=9$. Then, by Theorem 3.5, the optimal sparsity is $9+2(4-1)=15$. The following matrices are frame matrices with respect to a given orthonormal basis of two different unit-norm tight frames in $\mathbb{R}^{4}$ :

and

$$
\Phi_{1}=\left[\begin{array}{ccccccccc}
1 & 1 & \sqrt{\frac{1}{8}} & \sqrt{\frac{1}{8}} & 0 & 0 & 0 & 0 & 0 \\
0 & 0 & \sqrt{\frac{7}{8}} & -\sqrt{\frac{7}{8}} & \sqrt{\frac{1}{4}} & \sqrt{\frac{1}{4}} & 0 & 0 & 0 \\
0 & 0 & 0 & 0 & \sqrt{\frac{3}{4}} & -\sqrt{\frac{3}{4}} & \sqrt{\frac{3}{8}} & \sqrt{\frac{3}{8}} & 0 \\
0 & 0 & 0 & 0 & 0 & 0 & \sqrt{\frac{5}{8}} & -\sqrt{\frac{5}{8}} & 1
\end{array}\right]
$$

$$
\Phi_{2}=\left[\begin{array}{ccccccccc}
1 & \sqrt{\frac{5}{8}} & \sqrt{\frac{5}{8}} & 0 & 0 & 0 & 0 & 0 & 0 \\
0 & \sqrt{\frac{3}{8}} & -\sqrt{\frac{3}{8}} & \sqrt{\frac{3}{8}} & \sqrt{\frac{3}{8}} & \sqrt{\frac{3}{8}} & \sqrt{\frac{3}{8}} & 0 & 0 \\
0 & 0 & 0 & \sqrt{\frac{5}{8}} & -\sqrt{\frac{5}{8}} & 0 & 0 & 1 & 0 \\
0 & 0 & 0 & 0 & 0 & \sqrt{\frac{5}{8}} & -\sqrt{\frac{5}{8}} & 0 & 1
\end{array}\right] .
$$


Clearly, both frame matrices satisfy $\left\|\Phi_{1}\right\|_{0}=\left\|\Phi_{2}\right\|_{0}=15$, hence the corresponding frames are optimally sparse in $\mathscr{F}(4,9)$. We remark that $\Phi_{1}$ is the frame matrix of $\operatorname{STTF}(4,9)$.

Remark 3.8 Tight frames have the special property that their spectrum uniquely defines the frame operator. While it is shown in Casazza et al. (2011b) that given a diagonal frame operator a variant of Algorithm 1 for non-tight frames always constructs the optimally sparse frame associated to that operator, there may exist a sparser frame associated with a non-diagonal frame operator having the same spectrum. Hence in such cases spectral tetris does not always find the sparsest frame of a given spectrum.

Remark 3.9 For redundancy smaller than 2, the picture is less clear. It has been shown by Casazza et al. (2013) and Lemvig et al. (2013) that the spectral tetris algorithm works even in certain cases where $m<2 n$. However, to our knowledge, a systematic analysis of optimal sparsity for low redundancy has not been performed.

\section{Sparse Dual Frames}

In this section we will take a different viewpoint regarding sparsity, and we now ask how sparse a dual frame of a given, fixed frame can be. This viewpoint is motivated by the fact that in many cases the decomposition frame $\left(\varphi_{i}\right)_{i=1}^{m}$ is given by the application at hand, e.g., by the way of measuring the data. In these situations, one is then interested in choosing a good dual frame of $\left(\varphi_{i}\right)_{i=1}^{m}$ for the reconstruction process. Notice again how the redundancy of frames plays a key role here since in the non-redundant case we would only have one option for exact reconstruction.

Again, the goal will be to achieve sparsity in the sense of Definitions 3.1, with the goal of allowing for efficient matrix-vector multiplication, just this time for the dual frame (Note that in contrast to the previous section, the basis used for representing the dual frame is the same as for the frame at hand and hence fixed). In other words, we are interested in the following minimization problem:

$$
\min \|\Psi\|_{0} \quad \text { s.t. } \quad \Phi \Psi^{*}=I_{n},
$$

where the frame $\Phi$ is given. In line with Definition 3.2, we name the solutions of (4.1) optimally sparse duals; note that as for optimally sparse frames, these solutions are, in general, not unique. In the following two subsections we will focus on analyzing the possible values of the objective function $\|\Psi\|_{0}$ in (4.1), and in the final subsection we will comment on the problem of finding the minimizers.

\subsection{Optimal Sparsity of Dual Frames}

In this subsection we investigate the possible sparsity levels in the set of all dual frames. We start with a simple upper bound.

Lemma 4.1 (Li et al. (2013); Krahmer et al. (2013)) Suppose $\Phi$ is a frame for $\mathbb{R}^{n}$. Then there exists a dual frame $\Psi$ of $\Phi$ with

$$
\|\Psi\|_{0} \leq n^{2} .
$$


Proof Let $J \subset\{1,2, \ldots, m\}$ be such that $\# J=n$ and the corresponding frame vectors $\left(\varphi_{i}\right)_{i \in J}$ are linearly independent. Such a set always exists, as in order to form a frame, the columns of $\Phi$ must span $\mathbb{R}^{n}$. Let $\left(\psi_{i}\right)_{i \in J}$ be the unique (bi-orthogonal) dual of $\left(\varphi_{i}\right)_{i \in J}$, and set $\psi_{i}=0$ for $i \notin J$. Then we obviously have $\|\Psi\|_{0} \leq n^{2}$.

Without additional assumptions on the frame, only the trivial lower bound $\|\Psi\|_{0} \geq$ $n$ on the dual frame sparsity can be established (see Li et al. (2013)); this bound is achieved if and only if the frame contains the canonical basis vectors. To precisely determine the optimal sparsity in the set of all duals of a given frame, we thus need to take further properties of the frame into account. In particular, the spark of the frame matrix plays an important role. Recall that the spark of a matrix $\Phi \in \mathbb{R}^{n \times m}$ is defined as the smallest number of linearly dependent columns of $\Phi$ and is denoted by spark $(\Phi)$. For an invertible $n \times n$ matrix $\Phi$, one sets spark $(\Phi)=n+1$. In fact, we will need the following refined version of the spark.

Definition 4.2 Let $\Phi \in \mathbb{R}^{n \times m}$. Then $\operatorname{spark}_{j}(\Phi)$ denotes the size of the smallest set $I \subset\{1, \ldots, m\}$ of column indices such that the columns with indices in $I$ of the matrix $\Phi$ are linearly independent while the columns with indices in $I$ of the $(n-1) \times m$ submatrix $\Phi^{(j)}$ of $\Phi$ with the $j$ th row deleted are linearly dependent.

We can now state the main result of this subsection.

Theorem 4.3 (Krahmer et al. (2013)) Suppose $\Phi$ is a frame for $\mathbb{R}^{n}$. Then the optimally sparse dual frame $\Psi$ of $\Phi$ satisfies

$$
\|\Psi\|_{0}=\sum_{j=1}^{n} \operatorname{spark}_{j}(\Phi) .
$$

Proof Let $\Psi$ be an optimally sparse dual of $\Phi$. Fix $j \in\{1, \ldots, n\}$, and let $\varphi_{k}^{(j)}$ denote the $k$ th column of $\Phi^{(j)}$. Since $\Phi$ and $\Psi$ are assumed to be dual frames, we have that $\Phi^{(j)}\left(\psi^{j}\right)^{*}=0_{n-1}$ holds, where $\psi^{j}$ denotes the $j$ th row of $\Psi$ and $0_{n-1}$ the zero vector in $\mathbb{R}^{n-1}$. This shows that $\left(\varphi_{k}^{(j)}\right)_{k \in \operatorname{supp} \psi^{j}}$ must be linearly dependent. On the other hand, the frame vectors $\left(\varphi_{k}\right)_{k \in \operatorname{supp} \psi^{j}}$ must be linearly independent, as otherwise one of these columns would be a linear combination of the others, which would allow for the construction of $\tilde{\psi}^{j}$ with supp $\tilde{\psi}^{j} \subsetneq \operatorname{supp} \psi^{j}$ such that $\Phi\left(\tilde{\psi}^{j}\right)^{*}=e_{j}$. This in turn would imply that the frame, whose frame matrix is obtained from $\Psi$ by replacing the row $\psi^{j}$ by $\tilde{\psi}^{j}$ is also a dual frame of $\Phi$, so $\Psi$ is not the optimally sparse dual, contradicting our assumption. Therefore we obtain $\left|\operatorname{supp} \psi^{j}\right| \geq \operatorname{spark}_{j}(\Phi)$ which, in turn, implies that $\|\Psi\|_{0} \geq \sum_{j=1}^{n} \operatorname{spark}_{j}(\Phi)$.

To complete the proof we need to show existence of a dual frame that meets this lower bound. To that intent, fix $j \in\{1, \ldots, n\}$, and let $S$ be a set of $\operatorname{size}^{\operatorname{spark}_{j}}(\Phi)$ such that $\left(\varphi_{k}\right)_{k \in S}$ is a set of linearly independent columns of $\Phi$ such that the corresponding columns of $\Phi^{(j)}$ are linearly dependent. That is, there exist $\left(\lambda_{k}\right)_{k \in S}$ such that $\sum_{k \in S} \lambda_{k}\left(\varphi^{(j)}\right)_{k}=0$, but $\left(\sum_{k \in S} \lambda_{k} \varphi_{k}\right)_{j}=a \neq 0$. This leads us to define $\psi^{j}$ via

$$
\psi_{k}^{j}= \begin{cases}\frac{\lambda_{k}}{a} & \text { if } k \in S, \\ 0 & \text { if } k \in\{1, \ldots, m\} \backslash S,\end{cases}
$$


as this definition yields $\Phi\left(\psi^{j}\right)^{*}=e_{j}$. Therefore, the matrix $\Psi$ with rows $\psi^{j}, j \in$ $\{1, \ldots, n\}$, corresponds to a dual frame which is $\sum_{j=1}^{n} \operatorname{spark}_{j}(\Phi)$-sparse.

By definition we have $\operatorname{spark}_{j}(\Phi) \geq \operatorname{spark}\left(\Phi^{(j)}\right)$ for every $j=1, \ldots, n$. Hence, we immediately have the following useful corollary of Theorem 4.3.

Corollary 4.4 Suppose $\Phi$ is a frame for $\mathbb{R}^{n}$. Then any dual frame $\Psi$ of $\Phi$ satisfies

$$
\|\Psi\|_{0} \geq \sum_{j=1}^{n} \operatorname{spark}\left(\Phi^{(j)}\right) .
$$

\section{$4.2 n^{2}$-Sparse Duals}

By Lemma 4.1 we know that it is always possible to find a dual frame with sparsity level $n^{2}$. The result below states that for a large class of frames this is actually the best, or rather the sparsest, one can achieve. Recall that an $n \times m$ matrix is said to be in general position if any sub-collection of $n$ (column) vectors is linearly independent, that is, if any $n \times n$ submatrix is invertible. Such matrices are sometimes called full sparkframes, such as in Alexeev et al. (2012), since their spark is maximal, i.e., $n+1$.

Theorem 4.5 (Krahmer et al. (2013)) Suppose $\Phi$ is a frame for $\mathbb{R}^{n}$ such that the submatrix $\Phi^{(j)}$ is in general position for every $j=1, \ldots, n$. Then any dual frame $\Psi$ of $\Phi$ satisfies

$$
\|\Psi\|_{0} \geq n^{2} .
$$

In particular, the optimally sparse dual satisfies $\|\Psi\|_{0}=n^{2}$

Proof Since $\Phi^{(j)}$ is in general position, we have spark $\left(\Phi^{(j)}\right)=n$ for each $j=$ $1, \ldots, n$. By Corollary 4.4, this implies (4.2). Finally, by Lemma 4.1, we can conclude that the optimally sparse dual frame satisfies $\|\Psi\|_{0}=n^{2}$.

Remark 1 The proof of Theorem 4.3 entails that for a frame $\Phi$ in general position, every support set consisting of $n$ locations in each of the $n$ rows of the dual frame matrix is realized by a dual frame of $\Phi$. Thus a way to find $n^{2}$-sparse duals of such a frame is to fix a sparsity pattern (for example at random) and find a dual frame supported in this set row by row via inverting $n \times n$ submatrices of $\Phi$. This approach has been introduced and discussed in Li et al. (2013) under the name zero-forcing. As an alternative approach to find $n^{2}$-sparse dual frames, Li et al. (2013) also propose $\ell_{1}$-minimization.

We now illustrate Theorem 4.5 with a number of examples of frames which are well-known to be in general position, and which thus do not allow for dual frames with less than $n^{2}$ non-vanishing entries. 
Example 4.6 For any $n, m \in \mathbb{N}$ with $m \geq n$, let $a_{i}>0, i=1, \ldots, n$ with $a_{i} \neq a_{j}$ for all $i \neq j$, and let $b_{j} \in \mathbb{R}, j=1, \ldots, m$ with $b_{j} \neq b_{i}, j \neq i$. Generalized Vandermonde frames are defined by:

$$
\Phi=\left[\begin{array}{cccc}
a_{1}^{b_{1}} & a_{1}^{b_{2}} & \cdots & a_{1}^{b_{m}} \\
\vdots & \vdots & & \vdots \\
a_{n}^{b_{1}} & a_{n}^{b_{2}} & \cdots & a_{n}^{b_{m}}
\end{array}\right] .
$$

It is well-known, see e.g., Gantmacher $(1959, \S 8.1)$, that the submatrix $\Phi^{(j)}$ is in general position for every $j=1, \ldots, m$, which, by Theorem 4.5 , implies that optimally sparse duals of $\Phi$ are $n^{2}$-sparse.

As previously mentioned the results in this paper also hold for complex frames. The next two examples are frames for $\mathbb{C}^{n}$ whose sparsest dual has sparsity $n^{2}$.

Example 4.7 Let $n \in \mathbb{N}$, and let $m$ be prime. Let $\Phi$ be a partial FFT (Fast Fourier Transform) matrix of size $n \times m$, that is, $\Phi$ is constructed by picking $n$ rows of an $m \times m$ DFT (Discrete Fourier Transform) matrix at random. Recall that the $m \times m$ DFT matrix is defined by $\left(e^{-2 \pi i j k / m}\right)_{0 \leq j, k \leq m-1}$. We remark that $\Phi$ is a tight frame. Moreover, any $\Phi^{(j)}$ is in general position, as the determinant of any $(n-1) \times(n-1)$ submatrix of $\Phi$ is non-zero which is a consequence of Chebotarev theorem about roots of unity stating that any minor of an $m \times m$ DFT matrix is non-zero whenever $m$ is prime; see Stevenhagen and Lenstra (1996) and Pakovich (2007). Our conclusion is again that the sparsest dual frame $\Psi$ of $\Phi$ satisfies $\|\Psi\|_{0}=n^{2}$.

Example 4.8 For $n \in \mathbb{N}$ prime and $m=n^{2}$, Krahmer et al. (2008) showed that for almost every $g \in \mathbb{C}^{n}$, the Gabor frame with $n$ time bins and $n$ frequency bins generated by $g$ (see Section 4.3) has a frame matrix with no zero minors. For this reason, the optimally sparse duals of such Gabor frames have sparsity $n^{2}$.

In fact, the property of having a sparsest dual with sparsity $n^{2}$ is a very generic property in the sense that the set $\mathscr{N}(n, m)$ of all frames of $m$ vectors in $\mathbb{R}^{n}$ whose sparsest dual has $n^{2}$ non-zero entries contains an open, dense subset in $\mathbb{R}^{n \times m}$ and has a compliment of measure zero. Let $\mathscr{P}(n, m)$ be the set of all frames $\Phi$ which satisfy spark $\left(\Phi^{(j)}\right)=n$ for all $j=1, \ldots, n$. By Corollary 4.4 we have that $\mathscr{P}(n, m) \subset$ $\mathscr{N}(n, m)$. We are now ready to state the result saying that "most" frames have a sparsest dual with sparsity level $n^{2}$.

Lemma 4.9 Suppose $m \geq n$. Then the set $\mathscr{P}(n, m)$ is open and dense in $\mathbb{R}^{n \times m}$, and $\mathscr{P}(n, m)^{c}$ is of measure zero.

Proof Note that $\Phi=\left[x_{k, \ell}\right]_{k \in\{1, \ldots, n\}, \ell \in\{1, \ldots, m\}} \in \mathscr{P}(n, m)$ if and only if $\Phi$ has full-rank and the polynomials in $x_{k, \ell}$ given by $\operatorname{det}\left(\left[\Phi^{(j)}\right]_{I}\right)$ are non-zero for each $j \in\{1, \ldots, m\}$ and $I \in S$, where $S$ is the collection of all subsets of $\{1, \ldots, m\}$ of size $n-1$. Here $\left[\Phi^{(j)}\right]_{I} \in \mathbb{R}^{(n-1) \times(n-1)}$ denotes the matrix $\Phi^{(j)}$ restricted to the columns in the index set $I$. This shows that $\mathscr{P}(n, m)$ is open in the Zariski topology ${ }^{1}$. Since the set $\mathscr{P}(n, m)$

\footnotetext{
1 For the definition of the Zariski topology, we refer to the book by Hartshorne (1977).
} 
is non-empty by Example 4.6, it is thereby open and dense in the standard topology, see Alexeev et al. (2012). Finally, since $\mathscr{P}(n, m)^{c}$ is a proper subset and closed in the Zariski topology, it is of measure zero.

By Lemma 4.9, we can conclude that any frame of $m$ vectors in $\mathbb{R}^{n}$ is arbitrarily close to a frame in $\mathscr{N}(n, m)$.

Theorem 4.10 (Krahmer et al. (2013)) Every frame is arbitrarily close to a frame whose sparsest dual $\Psi$ satisfies $\|\Psi\|_{0}=n^{2}$.

Another consequence of Lemma 4.9 is that for many randomly generated frames, the sparsest dual has sparsity level $\|\Psi\|_{0}=n^{2}$. As an example, this holds when the entries of $\Phi$ are drawn independently at random from a standard normal distribution or when the frame is obtained by a small Gaussian random perturbation of a given $n \times m$ matrix.

\subsection{Sparse Gabor dual frames of Gabor frames}

A special case studied by Li et al. (2013) is the scenario that both the frame and its dual frame are Gabor frames. To define a Gabor frame, denote by $S: \mathbb{C}^{n} \rightarrow \mathbb{C}^{n}$ the shift operator given by $\left(z_{0}, \ldots, z_{n-1}\right)^{t} \mapsto\left(z_{1}, \ldots, z_{n-1}, z_{0}\right)^{t}$ and by $M: \mathbb{C}^{n} \rightarrow \mathbb{C}^{n}$ the modulation operator given by $\left(z_{0}, \ldots, z_{n-1}\right)^{t} \mapsto\left(z_{0}, e^{2 \pi i / n} z_{1}, \ldots, e^{2 \pi i(n-1) / n} z_{n-1}\right)^{t}$. Then for $p, q$ both divisors of $n$, the discrete Gabor system with $q$ time bins and $p$ frequency bins generated by a vector $g \in \mathbb{C}^{n}$ is given by $\left(M^{j \omega} S^{k a} g\right)_{j, k=1}^{p, q}$, where $\omega=\frac{n}{p}$ and $a=\frac{n}{q}$. If such a system is a frame for $\mathbb{C}^{n}$, it is called a Gabor frame (Wexler and Raz, 1990; Gröchenig, 2001). Gabor frames are of great importance for signal processing, as the Gabor frame coefficients can be interpreted as the local frequency content of a signal. As it turns out, a Gabor frame always has a Gabor dual, that is, a frame that is also a Gabor frame; if $m=p q>n$, they have even infinitely many Gabor duals. The generator $\gamma \in \mathbb{C}^{n}$ of a dual Gabor frame is called a dual Gabor window.

A main result of Li et al. (2013) gives upper and lower bounds for the sparsity of Gabor duals of Gabor frames. Here the relevant quantity is the sparsity of the dual frame generator as it directly determines the sparsity of the dual frame. Then the sparsity bounds are as follows.

Theorem 4.11 (Li et al. (2013)) Let $\Phi$ be the Gabor frame with $q$ time bins and $p$ frequency bins generated by $g \in \mathbb{C}^{n}$. The sparsest dual Gabor window $\gamma \in \mathbb{C}^{n}$ satisfies

$$
\frac{n}{q} \leq\|\gamma\|_{0} \leq \frac{n^{2}}{p q}
$$

and

$$
n p \leq\|\Psi\|_{0} \leq n^{2},
$$

where $\Psi=\left(M^{j \omega} S^{k a} \gamma\right)_{j, k=1}^{p, q}$. 
Proof The dual frames of $\Phi$ that have Gabor structure are characterized by the Wexler-Raz relations from Wexler and Raz (1990), which states that two generators $g$ and $\gamma$ are a pair of dual Gabor windows if and only if

$$
\frac{p q}{n}\left\langle\gamma, M^{j q} S^{k p} g\right\rangle=\delta_{j} \delta_{k} \quad \text { for } j=0, \ldots, \omega-1 \text { and } k=0, \ldots, a-1 .
$$

These equations can be rewritten as a set of $a$ linear systems of the form

$$
G_{i} \Gamma_{i}=\frac{1}{p} e_{1}, \quad i=0, \ldots, a-1,
$$

where

$$
G_{i}=\left[\begin{array}{cccc}
g_{i} & g_{i+a} & \cdots & g_{i+a(q-1)} \\
g_{i+p} & g_{i+a+p} & \cdots & g_{i+a(q-1)+p} \\
\vdots & \vdots & \ddots & \vdots \\
g_{i+p(\omega-1)} & g_{i+a+p(\omega-1)} & \cdots & g_{i+a(q-1)+p(\omega-1)}
\end{array}\right] \quad \text { and } \quad \Gamma_{i}=\left[\begin{array}{c}
\bar{\gamma}_{i} \\
\bar{\gamma}_{i+a} \\
\vdots \\
\bar{\gamma}_{i+a(q-1)}
\end{array}\right]
$$

We refer to the proof of Theorem 3.3 by Li et al. (2013) for the details of the above derivation. Since each of the linear systems $G_{i} \Gamma_{i}=\frac{1}{p} e_{1}, i=0, \ldots, a-1$, consists of $\omega$ equations with $q$ unknowns, the sparsest solution to $G_{i} \Gamma_{i}=\frac{1}{p} e_{1}$ has sparsity between 1 and $\omega$, that is, $1 \leq\left\|\Gamma_{i}\right\|_{0} \leq \omega$. Since $\|\gamma\|_{0}=a\left\|\Gamma_{i}\right\|_{0}$ and $\|\Psi\|_{0}=p q\|\gamma\|_{0}$, the two estimates (4.3) and (4.4) follow.

Example 4.8 shows that when $n \in \mathbb{N}$ is prime, and one has $n$ time bins as well as $n$ frequency bins, the statement of Theorem 4.11 holds for almost all generators $g \in \mathbb{C}^{n}$. Note that in this case the lower and upper bound agree, hence the result is sharp.

For sparsity to be interpreted as localization in the application scenario, the nonzero entries of the generator are required to be located in adjacent positions. We say that supp $g$ is connected to refer to this property of a generator $g$. The next result shows that if this is the case, then there exists a dual Gabor window $\gamma$ that meets the lower sparsity bound of Theorem 4.11.

Theorem 4.12 (Li et al. (2013)) Let $\Phi$ be the Gabor frame with $q$ time bins and $p$ frequency bins generated by $g \in \mathbb{C}^{n}$. Suppose that supp $g$ is connected and $a \leq$ $\#\{\operatorname{supp} g\} \leq q$. Then the sparsest dual Gabor window $\gamma \in \mathbb{C}^{n}$ satisfies $\|\gamma\|_{0}=a$ and $\|\Psi\|_{0}=n q$, where $\Psi=\left\{M^{j \omega} S^{k a} \gamma\right\}_{j, k=1}^{p, q}$.

Proof Since supp $g$ is connected and $a \leq \#\{\operatorname{supp} g\} \leq q$, each column of $G_{i}$ has at most one non-zero entry. Moreover, at least one of the entries in the first row of $G_{i}$ is non-zero; let $k$ be the corresponding column index. Then $\Gamma_{i}=\left(p \cdot g_{i+a(k-1)}\right)^{-1} e_{k}$ is a one-sparse solution to $G_{i} \Gamma_{i}=\frac{1}{p} e_{1}$ for each $i=0, \ldots, a-1$.

Comparable results can be derived for other regimes of the parameters and other support sizes of $g$. For details, we refer the reader to Li et al. (2013). 


\section{Discussion and Future Directions}

Comparing the problems discussed in Sections 3 and 4, one observes that the corresponding optimally sparse solutions behave very differently. For optimally sparse frames, a large fraction of the entries vanishes (only between $m$ and $2 m$ entries will not vanish), hence there is a limited number of degrees of freedom and the resulting frames are structurally very similar. For optimally sparse duals, in contrast, the sparsity can vastly vary. For duals of frames in general position, the minimal number of non-vanishing entries is $n^{2}$ and the minimizer, in general, non-unique. As a consequence, there is a large number of degrees of freedom that allows for structurally very different solutions. For example, the $n^{2}$ non-vanishing entries could be concentrated in few columns or evenly distributed over all columns.

Consequently, potential future research directions relating to the two problems are rather different:

For sparse frames, we understand the cases presented above where $m \geq 2 n$ quite well. But as mentioned in Remark 3.9, the low redundancy cases are open. Also, a more general question can be posed, namely the design of sparse frames with additional design specifications. One example for such specifications is low coherence, which was used as a design criteria for Steiner equiangular tight frames in the work of Fickus et al. (2012).

Regarding sparse duals, we have a complete characterization of the achievable sparsity levels, but the question remains which of the many structurally different optimally sparse duals is best from a computational viewpoint. As mentioned above, a dual frame with all $n^{2}$ non-vanishing entries contained in a few columns discards all of the redundant information, so intuitively it is less desirable than distributing the entries evenly over the columns. Hence it is important for a complete understanding to quantify this preference for one optimally sparse dual frame over the other in order to decide between two duals in case such an intuitive judgment is not possible. As the number of possible optimally sparse duals is very large, a satisfactory answer to this question must also involve algorithms to find duals which are at least close to the desired optimum. Furthermore, it would be interesting to find concrete examples of frame classes that allow for duals which are considerably sparser than in the generic case.

Acknowledgements The authors would like to thank the referees whose detailed reports have significantly improved the presentation of the paper.

The second author acknowledges support by the Einstein Foundation Berlin, by Deutsche Forschungsgemeinschaft (DFG) Grant SPP-1324 KU 1446/13 and DFG Grant KU 1446/14, by the DFG Collaborative Research Center TRR 109 "Discretization in Geometry and Dynamics", and by the DFG Research Center MATHEON "Mathematics for Key Technologies" in Berlin.

\section{References}

Alexeev B, Cahill J, Mixon DG (2012) Full spark frames. J. Fourier Anal. Appl., 18(6):1167-1194 
Bodmann BG, Casazza PG, Kutyniok G (2011) A quantitative notion of redundancy for finite frames. Appl. Comput. Harmon. Anal., 30(3):348-362.

Calderbank R, Casazza PG, Heinecke A, Kutyniok G, Pezeshki A (2011) Sparse fusion frames: existence and construction. Adv. Comput. Math., 35(1):1-31.

Candès EJ, Romberg JK, Tao T (2006) Stable signal recovery from incomplete and inaccurate measurements. Comm. Pure Appl. Math., 59(8):1207-1223.

Casazza PG, Fickus M, Mixon DG, Wang Y, Zhou Z (2011a) Constructing tight fusion frames. Appl. Comput. Harmon. Anal., 30(2):175-187.

Casazza PG, Heinecke A, Kornelson K, Wang Y, Zhou Z (2013) Necessary and sufficient conditions to perform Spectral Tetris. Linear Algebra Appl., 438(5):22392255.

Casazza PG, Heinecke A, Krahmer F, Kutyniok G (2011b) Optimally sparse frames. IEEE Trans. Inform. Theory, 57(11):7279-7287.

Casazza PG, Kutyniok G, editors (2013) Finite frames: Theory and applications. Applied and Numerical Harmonic Analysis. Birkhäuser/Springer, New York.

Christensen O (2008) Frames and bases: An introductory course.. Applied and Numerical Harmonic Analysis. Birkhäuser Boston Inc., Boston, MA.

Daubechies I, Grossmann A, Meyer Y (1986) Painless nonorthogonal expansions. J. Math. Phys., 27(5):1271-1283.

Donoho DL (2006) Compressed sensing. IEEE Trans. Inform. Theory, 52(4):12891306.

Duffin RJ and Schaeffer AC (1952) A class of nonharmonic Fourier series. Trans. Amer. Math. Soc., 72:341-366.

Eldar YC, Kutyniok G, editors (2012) Compressed sensing: Theory and applications. Cambridge University Press, Cambridge.

Fickus M, Mixon DG, Tremain JC (2012) Steiner equiangular tight frames. Linear Algebra Appl., 436(5):1014-1027.

Gantmacher FR (1959) Applications of the theory of matrices. Translated by J. L. Brenner, with the assistance of D. W. Bushaw and S. Evanusa. Interscience Publishers, Inc., New York.

Gröchenig K (2001) Foundations of time-frequency analysis. Applied and Numerical Harmonic Analysis. Birkhäuser Boston Inc., Boston, MA.

Hartshorne R (1977) Algebraic geometry. Springer-Verlag, New York. Graduate Texts in Mathematics, No. 52.

Krahmer F, Kutyniok G, Lemvig J (2013) Sparsity and spectral properties of dual frames. Linear Algebra Appl., 439(4):982-998.

Krahmer F, Pfander GE, Rashkov P (2008) Uncertainty in time-frequency representations on finite abelian groups and applications. Appl. Comput. Harmon. Anal., 25(2):209-225.

Kutyniok G (2013) Theory and applications of compressed sensing. GAMM Mitteilungen, to appear.

Kutyniok G, Okoudjou KA, Philipp F, Tuley EK (2013) Scalable frames. Linear Algebra Appl., 438(5):2225-2238.

Lemvig J, Miller C, Okoudjou KA (2013) Prime tight frames. Adv. Comput. Math., to appear, DOI: 10.1007/s10444-013-9309-0. 
Li S (1995) On general frame decompositions. Numer. Funct. Anal. Optim., 16(910):1181-1191.

Li S, Liu Y, Mi T (2013) Sparse dual frames and dual Gabor functions of minimal time and frequency supports. J. Fourier Anal. Appl., 19(1):48-76.

Pakovich F (2007) A remark on the Chebotarev theorem about roots of unity. Integers, 7:A18, 2.

Stevenhagen P, Lenstra, Jr. HW (1996) Chebotarëv and his density theorem. Math. Intelligencer, 18(2):26-37.

Wexler J, Raz S (1990) Discrete gabor expansions. Signal Process., 21(3):207-220. 